\section{Controllability of Quantum Harmonic Oscillators}

\author{
Mazyar Mirrahimi and Pierre Rouchon
}

\begin{abstract}
It is proven in a previous paper that any modal approximation of the one-dimensional quantum harmonic oscillator is controllable. We prove here that, contrary to such finite-dimensional approximations, the original infinite-dimensional system is not controllable: Its controllable part is of dimension 2 and corresponds to the dynamics of the average position. More generally, we prove that, for the quantum harmonic oscillator of any dimension, similar lacks of controllability occur whatever the number of control is: the controllable part still corresponds to the average position dynamics. We show, with the quantum particle in a moving quadratic potential, that some physically interesting motion planning questions can be however solved.
\end{abstract}

Index Terms-Nonlinear controllability, quantum systems, Schrödinger equation.

\section{INTRODUCTION}

Schirmer et al. have considered for any integer $n>0$, the following controlled Schrödinger equation (state $\psi \in \mathbb{C}^{n+1}$ and control $u \in \mathbb{R}$ )

$$
\imath \frac{d}{d t} \psi=\left(H_{0}+u H_{1}\right) \psi
$$

where $H_{0}$ and $H_{1}$ are the following $(n+1) \times(n+1)$ Hermitian matrices:

$$
\begin{aligned}
H_{0} & =\left(\begin{array}{ccccc}
\frac{1}{2} & 0 & & \cdots & 0 \\
0 & \frac{3}{2} & 0 & & \\
& 0 & \frac{5}{2} & 0 & \vdots \\
\vdots & & & \ddots & 0 \\
0 & & \cdots & 0 & \frac{2 n+1}{2}
\end{array}\right) \\
H_{1} & =\left(\begin{array}{cccccc}
0 & 1 & 0 & \cdots & & 0 \\
1 & 0 & \sqrt{2} & \ddots & & \vdots \\
0 & \sqrt{2} & 0 & \sqrt{3} & & \vdots \\
\vdots & & \sqrt{3} & & \ddots & 0 \\
\vdots & & \ddots & \ddots & & \sqrt{n+1} \\
0 & \cdots & & 0 & \sqrt{n+1} & 0
\end{array}\right) .
\end{aligned}
$$

Using the general controllability result of [8], they have proved that this system is controllable. Such a system is the truncation, up to the first $n+1$ eigenstates whose energies are between $1 / 2$ and $n+(1 / 2)$, of the harmonic oscillator

$$
\imath \frac{\partial \psi}{\partial t}=-\frac{1}{2} \frac{\partial^{2} \psi}{\partial q^{2}}+\left(\frac{1}{2} q^{2}-u(t) q\right) \psi
$$

where the probability amplitude $\psi(t, q) \in \mathbb{C}$ is a function of $q \in \mathbb{R}$ and $t$. We prove here that such system is not controllable: Its controllable part corresponds to the classical harmonic dynamics followed by the average position $\langle q\rangle=\int_{-\infty}^{+\infty}|\psi(t, q)|^{2} q d q$

$$
\frac{d^{2}}{d t^{2}}\langle q\rangle=-\langle q\rangle+u
$$

Manuscript received December 17, 2002; revised November 30, 2003. Recommended by Associate Editor P. D. Christofides.

The authors are with the Centre Automatique et Systèmes, Ecole des Mines de Paris, 75272 Paris cedex 06, France (e-mail: mazyar.mirrahimi@ensmp.fr; pierre.rouchon@ensmp.fr).

Digital Object Identifier 10.1109/TAC.2004.825966
For the $N$-dimensional approximation, the commutation $[q, p]=\imath$ does not hold, what results in a controllability Lie algebra $N^{2}$, while for the infinite-dimensional description the Lie algebra is of dimension 4.

In fact we provide the decomposition into the controllable part (the previous second-order equation) and its uncontrollable part (an autonomous Schrödinger equation). This decomposition extends directly to any arbitrary space dimension $n$ and control number $m$ :

$$
\imath \frac{\partial \psi}{\partial t}(t, q)=-\frac{1}{2} \Delta \psi+\frac{1}{2}(A q, q)_{\mathbb{R}^{n}} \psi-\sum_{i=1}^{m} u_{i}\left(b_{i}, q\right)_{\mathbb{R}^{n}} \psi
$$

where $\mathbb{R} \times \mathbb{R}^{n} \ni(t, q) \mapsto \psi(t, q) \in \mathbb{C}$ is the probability amplitude, $\Delta=\sum_{i=1}^{n}\left(\partial^{2}\right) /\left(\partial q_{i}^{2}\right)$ the Laplace operator in $\mathbb{R}^{n}, A$ is a symmetric matrix of order $n$, the $b_{i}$ 's are vectors of $\mathbb{R}^{n}$ and $(.,.) \mathbb{R}^{n}$ is the Euclidian product in $\mathbb{R}^{n}$.

Although such strong lack of controllability tends to indicate that severe obstructions to the control of harmonic oscillators exist, the situation is not as disappointing as one might think. Assume that $u$ corresponds to an acceleration $-u=\ddot{\varrho}$. Then, (1) can be interpreted as the Schrödinger equation of a particle in a non-Galilean frame admitting the acceleration $\ddot{\varrho}$. The control is then directly related the absolute position of this frame $\varrho$ and the physical interpretation becomes clear: the particle is trapped in a box described by the quadratic potential $q^{2} / 2$. This box is moving and the control is the position of the box. This configuration is very similar to a classical one: the slosh problem where a tank filled with liquid is moved. The quantum particle plays the role of the liquid with its surface waves. The quadratic potential corresponds then to the tank that contains the liquid. We have then partial controllability properties similar to those obtained in [7] for the slosh problem: for any initial $a$ and final position $b$ of the box $\varrho$ and any steady-state $\psi_{n}$ of energy $n+1 / 2$, we describe explicitly the control $t \mapsto u(t)$ steering (1) from box position $a$ with $\psi=\psi_{n}$ at $t=0$ to box position $b$ with $\psi=\exp (\imath \theta) \psi_{n}$ at $t=T(T>0$ is any transition time) where $\theta \in \mathbb{R}$ is some physically meaningless phase shift.

The note is organized as follows: next section deals with the one dimensional case and its decomposition into controllable and uncontrollable parts. In Section III, we consider the $n$-dimensional cases with $m$ controls. In Section IV, we consider the moving potential and solve by elementary computations some motion planning problems admitting a simple physical meaning.

Preliminary versions of these results can be found in [10], [9].

\section{OnE-Dimensional CASE}

Denote by $p=-\imath(\partial) /(\partial q)$ the momentum operator and by $q$ the multiplication by $q$. Then, (1) reads

$$
\imath \frac{d}{d t} \psi=\frac{1}{2}\left(p^{2}+q^{2}\right) \psi-u q \psi=H_{0} \psi+u H_{1} \psi
$$

where $H_{0}=(1 / 2)\left(p^{2}+q^{2}\right)$ and $H_{1}=-q$. Following [8], let us compute the Lie algebra generated by $\imath H_{0}$ and $\imath H_{1}$, using the standard commutation relations: $[q, p]=\imath,[q, F(p)]=\imath F^{\prime}(p)$, and $[F(q), p]=\imath F^{\prime}(q)$ for any function $F$. We have

$$
\left[H_{0}, H_{1}\right]=\imath p \quad\left[H_{0}, p\right]=-\imath q \quad\left[H_{1}, p\right]=-\imath .
$$

Thus, this Lie algebra is of dimension 4 and the system is not controllable.

In fact, the controllable part coincides with the dynamics of the average position $\langle q\rangle=\int_{-\infty}^{+\infty} q|\psi(t, q)|^{2} d q$. Its dynamics given by the 
classical Ehrenfest theorem (see, e.g., [6]) corresponds then to the classical oscillator

$$
\frac{d}{d t}\langle q\rangle=\langle p\rangle \quad \frac{d}{d t}\langle p\rangle=-\langle q\rangle+u
$$

which is trivially controllable.

The uncontrollable part corresponds to a Schrödinger dynamics without control. Consider the following change of independent variables $(t, q) \mapsto(t, z=q-\langle q\rangle)$. Then, the Schrödinger equation reads with $\psi(t, q)=\exp (\imath\langle p\rangle z) \phi(t, z)$

$$
\imath \frac{d}{d t} \phi=\left(P^{2} / 2+Z^{2} / 2\right) \phi+\left(\langle q\rangle^{2} / 2-\langle p\rangle^{2} / 2-u\langle q\rangle\right) \phi
$$

where $P \equiv \imath(\partial) /(\partial z)$ and $Z \equiv z$. The following phase change:

$$
\phi(t, z)=\exp \left(-\imath \int_{0}^{t}\left(\langle q\rangle^{2} / 2-\langle p\rangle^{2} / 2-u\langle q\rangle\right)\right) \varphi(t, z)
$$

(gauge transformation since $\phi$ and $\varphi$ represent the same physical system) yields

$$
\imath \frac{d}{d t} \varphi=\left(P^{2} / 2+Z^{2} / 2\right) \varphi .
$$

The dynamics of $\phi(t, q)$ can be decomposed into two parts, a controllable one of dimension two (3), an uncontrollable one of infinite dimension (4).

The aforementioned computations are classical (see, e.g., [1]). Less classical is the interpretation as decomposition into controllable and uncontrollable parts. It is the infinite dimensional analogue of decomposition for the nonlinear system of finite dimension $(d) /(d t) \xi=f(\xi, u)$ via a nonlinear change of coordinates $\xi \mapsto \chi$ (see, e.g., [4]) where

$$
\chi=\left(\chi_{1}, \chi_{2}\right) \quad \frac{d}{d t} \chi_{1}=g_{1}\left(\chi_{1}\right) \quad \frac{d}{d t} \chi_{2}=g_{2}\left(\chi_{1}, \chi_{2}, u\right) .
$$

The uncontrollable part corresponds then to the autonomous dynamics on $\chi_{1}$. The following question becomes then natural. Take the controlled Schrödinger equation (infinite-dimensional case) with general Hermitian operators $H_{0}$ and $H_{1}$

$$
\imath \frac{d}{d t} \psi=\left(H_{0}+\sum_{1}^{n} u_{i} H_{i}\right) \psi
$$

Assume that the Lie algebra spanned by the skew-Hermitian operators $H_{0} / \imath$ and $H_{1} / \imath$ is of finite dimension. Does there exists a decomposition into a finite-dimensional controllable part and an infinite-dimensional uncontrollable part, similar to the case $H_{0}=p^{2} / 2+q^{2} / 2, H_{1}=$ $-q$ ?

\section{III. $N$-DiMENSIONAL CASE}

In contrast to the control equation for the wavefunction, which is bilinear with drift, the control equations for the average position and momentum of a harmonic oscillator in an $n$-dimensional configuration space are linear, and hence standard results for linear control are applicable.

Consider (2). By the Ehrenfest theorem, we have

$$
\frac{d}{d t}\langle q\rangle=\langle p\rangle \quad \frac{d}{d t}\langle p\rangle=-A\langle q\rangle+B u
$$

where $B=\left(b_{1}, \ldots, b_{m}\right),\langle q\rangle \in \mathbb{R}^{n}$ and $\langle p\rangle \in \mathbb{R}^{n}$ are the average position and momentum. This finite-dimensional system is controllable as soon as the rank of its Kalman controllability matrix is $2 n$ [5]. This is equivalent to the rank of $\left(B, A B, \ldots, A^{n-1} B\right)$ equals $n$.
Consider the following change of variables $(t, q) \mapsto(t, z=q-$ $\langle q\rangle)$. Let

$$
\psi(t, q)=\exp \left(i(\langle p\rangle, z)_{\mathbb{R}^{n}}\right) \phi(t, z) .
$$

Then, (2) becomes

$$
\begin{aligned}
\imath \frac{d}{d t} \phi(t, z)=\left(\frac{P^{2}}{2}\right. & \left.+\frac{1}{2}(A Z, Z)\right) \phi \\
& +\left(\frac{1}{2}(A\langle q\rangle,\langle q\rangle)_{\mathbb{R}^{n}}-\frac{1}{2}\langle p\rangle^{2}-(B u,\langle q\rangle)_{\mathbb{R}^{n}}\right) \phi
\end{aligned}
$$

where $P=\imath\left((\partial) /\left(\partial q_{1}\right), \ldots,(\partial) /\left(\partial q_{n}\right)\right)$ and $Z=\left(z_{1}, \ldots, z_{n}\right)$. Now, we use a gauge transformation

$$
\begin{aligned}
\phi(t, z)=\exp \left(-\imath \int_{0}^{t}\left(\frac{1}{2}(A\langle q\rangle,\langle q\rangle)_{\mathbb{R}^{n}}\right.\right. & \\
& \left.\left.-\frac{1}{2}\langle p\rangle^{2}-(B u,\langle q\rangle)_{\mathbb{R}^{n}}\right) d t\right) \varphi(t, z)
\end{aligned}
$$

where $\varphi$ obeys an autonomous equation

$$
\imath \frac{d}{d t} \varphi=\left(\frac{P^{2}}{2}+\frac{1}{2}(A Z, Z)\right) \varphi .
$$

To summarize: The controllable part of (2) corresponds to the controllable part of the $2 n$-dimensional linear system (5). Its dimension is the double of the rank of its "Kalman controllability matrix" $\left(B, A B, \ldots, A^{n-1} B\right)$.

\section{Particle in a Moving Quadratic Potential BoX}

Take (1) and assume that $u=-\ddot{\varrho}$ with $\varrho$ the position of the non Galilean frame associated to $q$. From Section II, we consider the finitedimensional system

$$
\langle\ddot{q}\rangle=-\langle q\rangle+u \quad \ddot{\varrho}=-u .
$$

This system is controllable with $y=\varrho+\langle q\rangle$, the average position in the Galilean frame, as Brunovsky output [5]

$$
\langle q\rangle=-\ddot{y} \quad \varrho=y+\ddot{y} \quad u=\ddot{y}+y^{(4)} .
$$

Assume now that our goal is to steer the particle from one spatial location described by its average position $a$ at $t=0$ to another position $b$ at $t=T>0$. Assume that the initial state $\psi(0, t)$ is a steady-state $\psi_{n}(q)$ with energy $n+1 / 2, n \in \mathbb{N}$. Our goal is also to recover this energy and its corresponding state at the end of the motion: we just want to translate the particle leaving its energy unchanged at the end. To perform such motions in a smooth manner, it suffices, using flatness based motion planning methods [2], [3], to set

$$
u=\ddot{\varsigma}+\varsigma^{(4)}
$$

where $\varsigma$ is any $C^{4}$ time function such that $\varsigma(0)=a, \varsigma(T)=b$, and $\varsigma^{(i)}(0)=\varsigma^{(i)}(T)=0$, for $i=1,2,3,4$. One can take, for example

$$
\varsigma(t)=\frac{a\left(1-\frac{t}{T}\right)^{\alpha}+b\left(\frac{t}{T}\right)^{\alpha}}{\left(1-\frac{t}{T}\right)^{\alpha}+\left(\frac{t}{T}\right)^{\alpha}}, \quad t \in[0, T]
$$

with $\alpha \geq 4$. The decomposition into the controllable and uncontrollable parts, (3) and (4), implies that the solution of (1) with $\psi(0, q)=$ 
$\psi_{n}(q)$ and $u(t)=\ddot{\varsigma}(t)+\varsigma^{(4)}(t)$ is explicit. For $t \in[0, T]$, the solution $\psi(t, q)$ is equal to

$$
\begin{aligned}
\exp \left[-\imath\left(\frac{(2 n+1) t}{2}+\varsigma^{(3)}(q+\ddot{\varsigma})\right.\right. & \\
& \left.\left.+\int_{0}^{t}\left(\frac{\ddot{\varsigma}^{2}}{2}-\frac{\left(\varsigma^{(3)}\right)^{2}}{2}+\left(\ddot{\varsigma}+\varsigma^{(4)}\right) \ddot{\varsigma}\right)\right)\right] \psi_{n}(q+\ddot{\varsigma}) .
\end{aligned}
$$

This results directly from Section II because

$$
\langle q\rangle=-\ddot{\varsigma}\langle p\rangle=-\varsigma^{(3)}
$$

Since we have also $\varrho=\varsigma+\ddot{\varsigma}$, we conclude that such open-loop control steers the particle from the average position $a$ to $b$ without changing its energy. The transition time $T$ can be arbitrary small and the control $u$ could be very large. Using Section III, our motion planning method can be easily extended to $n$ particles in $n$ quadratic potential box moving simultaneously in the three-dimensional physical space.

\section{CONCLUDING REMARK}

The fact that the only controllable part corresponds to the "classical" dynamics of the average positions is a rather surprising result. Since harmonic oscillators appear naturally in quantum-electrodynamics, we wonder if such results cannot be a starting point to imagine the control of the quantum dynamics of an electromagnetic field. In a certain sense, the question is: what is the quantum analogue for the boundary control of a classical wave equation.

\section{ACKNOWLEDGMENT}

The authors would like to thank C. Le Bris and G. Turinici for interesting discussions and [11].

\section{REFERENCES}

[1] A. G. Butkovskiy and Y. I. Samoilenko, Control of Quantum-Mechnical Processes and Systems. Norwell, MA: Kluwer, 1984, vol. 56.

[2] M. Fliess, J. Lévine, Ph. Martin, and P. Rouchon, "Flatness and defect of nonlinear systems: Introductory theory and examples," Int. J. Control, vol. 61, no. 6, pp. 1327-1361, 1995.

[3] —, "A Lie-Bäcklund approach to equivalence and flatness of nonlinear systems," IEEE Trans. Automat. Contr., vol. 44, pp. 922-937, July 1999.

[4] A. Isidori, Nonlinear Control Systems, 2nd ed. New York: SpringerVerlag, 1989.

[5] T. Kailath, Linear Systems. Upper Saddle River, NJ: Prentice-Hall, 1980.

[6] A. Messiah, Quantum Mechanics. Amsterdam, The Netherlands: North-Holland, 1962, vol. I\&II

[7] N. Petit and P. Rouchon, "Dynamics and solutions to some control problems for water-tank systems," IEEE Automat. Contr., vol. 47, pp. 594-609, Apr. 2002.

[8] V. Ramakrishna, M. Salapaka, M. Dahleh, and H. Rabitz, "Controllability of molecular systems," Phys. Rev. A, vol. 51, no. 2, pp. 960-966, 1995.

[9] P. Rouchon, "Control of a quantum particle in a moving box," École des Mines de Paris, Centre Autom. Systèmes, Paris, France, Tech. Rep. A/325, Oct. 2002.

[10] — " "On the control of quantum oscillators," École des Mines de Paris, Centre Autom. Systèmes, Paris, France, Tech. Rep. A/320, June 2002.

[11] S. G. Schirmer, H. Fu, and A. I. Solomon, "Complete controllability of quantum systems," Phys. Rev. A, vol. 63, no. 063 410, 2001.

\section{Dissipativity Theory for Nonnegative and Compartmental Dynamical Systems With Time Delay}

Wassim M. Haddad, VijaySekhar Chellaboina, and Tanmay Rajpurohit

\begin{abstract}
Nonnegative and compartmental dynamical system models are derived from mass and energy balance considerations that involve dynamic states whose values are nonnegative. These models are widespread in engineering and life sciences and typically involve the exchange of nonnegative quantities between subsystems or compartments wherein each compartment is assumed to be kinetically homogeneous. However, in many engineering and life science systems, transfers between compartments are not instantaneous and realistic models for capturing the dynamics of such systems should account for material in transit between compartments. Including some information of past system states in the system model leads to infinite-dimensional delay nonnegative dynamical systems. In this note, we develop dissipativity theory for nonnegative dynamical systems with time delay using linear storage functionals with linear supply rates. These results are then used to develop general stability criteria for feedback interconnections of nonnegative dynamical systems with time delay.
\end{abstract}

Index Terms-Compartmental systems, dissipativity theory, feedback systems, linear storage functionals, linear supply rates, nonnegative systems, time delay.

\section{INTRODUCTION}

Modern complex engineering systems are highly interconnected and mutually interdependent, both physically and through a multitude of information and communication networks. By properly formulating these systems in terms of subsystem interaction and energy/mass transfer, the dynamical models of many of these systems can be derived from mass, energy, and information balance considerations that involve dynamic states whose values are nonnegative. Hence, it follows from physical considerations that the state trajectory of such systems remains in the nonnegative orthant of the state space for nonnegative initial conditions. Such systems are commonly referred to as nonnegative dynamical systems in the literature [1]-[3]. A subclass of nonnegative dynamical systems are compartmental systems [3]-[13]. Compartmental systems involve dynamical models that are characterized by conservation laws (e.g., mass and energy) capturing the exchange of material between coupled macroscopic subsystems known as compartments. Each compartment is assumed to be kinetically homogeneous; that is, any material entering the compartment is instantaneously mixed with the material of the compartment. A key physical limitation of such systems is that transfers between compartments are not instantaneous and realistic models for capturing the dynamics of such systems should account for material, energy, or information in transit between compartments [11], [14]. Hence, to accurately describe the evolution of the aforementioned systems, it is necessary to include in any mathematical model of the system dynamics some information of the past system states. This of course leads to (infinite-dimensional) delay dynamical systems [15]-[17].

Manuscript received November 12, 2002; revised July 15, 2003. Recommended by Associate Editor R. A. Freeman. This work was supported in part by the Air Force Office of Scientific Research under Grant F49620-03-1-0178 and by the National Science Foundation under Grant ECS-0133038.

W. M. Haddad and T. Rajpurohit are with the School of Aerospace Engineering, Georgia Institute of Technology, Atlanta, GA 30332-0150 USA (e-mail: wm.haddad@aerospace.gatech.edu; tanmay_rajpurohit@ae.gatech.edu).

V. Chellaboina is with the Mechanical and Aerospace Engineering, University of Missouri, Columbia, MO 65211 USA (e-mail: ChellaboinaV@ missouri.edu). Digital Object Identifier 10.1109/TAC.2004.825967 